\title{
Corpus
}

\section{Syntaxe et Corpus. Présentation}

\section{Michèle Oliviéri}

\section{(2) OpenEdition}

Journals

\section{Édition électronique}

URL : http://journals.openedition.org/corpus/1950

DOI : 10.4000/corpus.1950

ISSN : 1765-3126

\section{Éditeur}

Bases; corpus et langage - UMR 6039

\section{Édition imprimée}

Date de publication : 1 novembre 2010

Pagination : 7-20

ISSN : 1638-9808

\section{Référence électronique}

Michèle Oliviéri, « Syntaxe et Corpus. Présentation », Corpus [En ligne], 9 | 2010, mis en ligne le 04 juillet 2011, consulté le 07 septembre 2020. URL : http://journals.openedition.org/corpus/1950 ; DOI :

https://doi.org/10.4000/corpus. 1950 


\section{Syntaxe et Corpus}

Michèle OLIVIERI

Université Nice Sophia-Antipolis / CNRS

UMR 6039 « Bases, Corpus, Langage »

\section{Une polémique historique}

En matière de syntaxe, la notion de corpus fut très controversée et reste encore problématique. Avant Chomsky, les structuralistes utilisaient des corpus, mais le terme désignait alors un échantillon représentatif et homogène d'un aspect syntaxique particulier ou d'une langue donnée et était utilisé dans une perspective behaviouriste exlusivement empiriste et taxinomique. C'est contre ce type de «corpus » que Chomsky s'est prononcé lorsqu'il a commencé à développer la grammaire générative dès les années 50 . En effet, il a montré notamment que la langue ne pouvait se réduire à un nombre fini d'énoncés puisque la compétence du locuteur lui permettait de produire une infinité de phrases grammaticales :

[...] il est évident que l'ensemble des phrases grammaticales ne peut être assimilé à aucun corpus particulier d'énoncés, recueilli par le linguiste dans son travail d'enquête ${ }^{1}$.

En outre, la représentativité d'un échantillon de langue est à l'évidence particulièrement difficile à assurer, et relativement incompatible avec son homogénéité. Cependant, Chomsky n'a jamais exclu complètement la nécessité de recourir aux données primaires, comme «procédure de découverte », mais il s'est attaché à construire une théorie qui va au-delà du simple

1 Chomsky (1957: 15): «[...] it is obvious that the set of grammatical sentences cannot be identified with any corpus of utterances obtained by the linguist in his field work».

Corpus $\mathrm{n}^{\circ} 9$ 《La syntaxe de corpus / Corpus Syntax » 


\section{OLIVIERI}

observable et qui rende compte de la «nature générale du langage $\gg$. De ce point de vue,
la grammaire d'une langue doit projeter le corpus fini et toujours plus ou moins accidentel des énoncés observés sur l'ensemble (présumé infini) des phrases grammaticales ${ }^{2}$.

La compétence - aujourd'hui I-language - est définie par Chomsky comme permettant au locuteur de produire et de comprendre un nombre illimité de mots et de phrases de sa langue mais aussi - et surtout - de juger de leur acceptabilité. C'est ainsi que les jugements de grammaticalité ont été, et sont encore, au cœur des études générativistes, faisant de l'analyse syntaxique une discipline de l'introspection. En effet, l'objet de la grammaire générative étant de décrire la compétence, les interrogations portent sur la connaissance interne que le locuteur a de sa langue et donc cherchent à savoir ce qui se passe dans son cerveau, et qui n'est pas forcément attesté dans un corpus.

$\mathrm{Si}$ cette démarche se justifie pleinement dans ce cadre théorique, une des conséquences malheureuses en est que des discussions interminables sont apparues autour de quelques phrases artificiellement fabriquées et qui réapparaissent indéfiniment dans la littérature. Certaines sont devenues célèbres bien que n'ayant que peu de chances de figurer dans un énoncé spontané :

(1) L'homme que je crois que Jean pense qui viendra...

Qui crois-tu qui/*que téléphonera?

Il n'a pas été trouvé de livres.

Malgré cela, la plupart des générativistes continuent à utiliser cette méthode, avec toutes les difficultés qu'elle engendre, notamment lorsque l'on se penche sur la diachronie puisqu'il n'est pas possible d'interroger les locuteurs des siècles précédents.

2 Chomsky (1957: 15) : «Any grammar of a language will project the finite and somewhat accidental corpus of observed utterances to a set (presumably infinite) of grammatical utterances ». 
Les limites de cette démarche sont alors bien évidemment mises en avant par les détracteurs de Chomsky, qui ont beau jeu de dénoncer ces pratiques, pour discréditer les générativistes, ou pour défendre d'autres perspectives. De fait, la linguistique de corpus connait un essor considérable depuis quelques années. Il est vrai qu'un autre paramètre joue également en faveur de cette approche, qui est l'avènement de l'informatique et les possibilités qu'elle offre désormais, tant en matière de ressources que d'outils d'exploitation, et dont les linguistes de la majeure partie du $\mathrm{XX}^{\mathrm{e}}$ siècle ne disposaient pas.

\section{La question de la grammaticalité}

La recherche du I-Language, des manifestations de la Faculté de Langage, i.e. au sens de Chomsky (2005) du « composant de la biologie humaine qui entre dans l'usage et l'acquisition du langage », a donc conduit les générativistes à s'attacher principalement aux jugements de grammaticalité.

Or, tous ceux qui ont pratiqué les tests de grammaticalité savent que ces jugements diffèrent souvent d'un locuteur à l'autre, ou restent incertains. Ainsi, certaines phrases sont définitivement astérisquées par certains auteurs alors qu'elles sont parfaitement acceptables pour d'autres.

En outre, il a été remarqué à maintes reprises que, en ce qui concerne la langue orale, non normative, le jugement du locuteur n'est pas toujours fiable. Ainsi, certains rejettent catégoriquement une structure qu'ils emploient pourtant fréquemment mais sans en avoir conscience. Il est manifeste que les gens ne parlent pas comme ils le croient. Que faire alors d'un tel jugement? Un des exemples français les plus frappants est la phrase (2) qui est souvent astérisquée, notamment lorsqu'elle est mise en contraste avec des formes dialectales où le clitique sujet est obligatoire, mais qui correspond en réalité à la forme normale en français spontané.

(2) Marie elle dort.

Or, les analyses proposées se fondent justement sur ces jugements, ce qui laisse parfois le lecteur perplexe. Il faudrait alors pouvoir juger les jugements de grammaticalité, évaluer les 
M. OLIVIERI

évaluations, ce qui semble être a priori une mission impossible. Qu'est-ce qui relève d'une «erreur», du locuteur ou du linguiste, ou d'une variation dans la langue, voire d'une diglossie?

C'est alors que le corpus vient à la rescousse de l'introspection. C'est en effet le corpus qui peut attester d'une occurence qui, sans cela, ne serait pas prise en considération dans l'analyse. C'est encore le corpus qui peut permettre de mesurer la fréquence d'une structure et de comparer les formes entre elles. C'est enfin le corpus qui permet d'accéder à des langues qui ne sont plus parlées aujourd'hui.

\section{Grands corpus et variation linguistique}

Les dialectologues - dont je suis - ont une grande pratique des enquêtes de terrain et de la constitution de corpus. Le temps est heureusement révolu où «théorie» et «empirie » étaient dissociées, le travail de terrain constituant une tâche subalterne alors que les penseurs ne quittaient pas leur bureau. On sait aujourd'hui l'importance que revêt la collecte des matériaux et l'avantage qu'il y a à recueillir soi-même les données que l'on veut étudier ${ }^{3}$. Ainsi, le choix des témoins, l'élaboration des questionnaires, l'utilisation des différentes techniques, l'écoute et la transcription des données sont autant d'éléments déterminants dans la constitution du corpus et son exploitation scientifique. Car il y a un va-et-vient permanent entre l'étude des données et l'enquête de terrain. Si le corpus permet l'analyse, c'est le modèle, le point de vue qui détermine l'élaboration du corpus, de sorte que théorie et corpus sont indissociables.

Or, la technologie moderne offre aujourd'hui de nombreuses possibilités. Outre une meilleure qualité et une accessibilité accrue des enregistrements, elle permet également, grâce aux progrès de l'informatique, de constituer et d'exploiter de très grands corpus. Le corpus n'est désormais plus un « échantillon », représentatif ou non, mais un objet multiforme, construit, en modification permanente, interrogeable de diverses manières et dont la taille garantit la fiabilité (le fameux «more

3 Cf. notamment Dalbera (2002). 


\section{Syntaxe et corpus}

data, better data»). Même si, face à l'infinité des énoncés possibles, ce très grand corpus évolutif reste un objet fini auquel le linguiste doit imposer une clôture provisoire pour mener à bien telle ou telle analyse, la critique initiale de Chomsky envers les corpus semble donc moins fondée et il n'y a désormais aucune raison pour ne pas (ré-)concilier grammaire générative et linguistique de corpus. Rappelons d'ailleurs ce que Chomsky (1965) précisait déjà :

Il n'est pas nécessaire, évidemment, de supposer que les points de vue empiriste et rationaliste peuvent toujours être strictement distingués et que ces courants ne peuvent se confondre. [...] Il est possible $[\ldots]$ de les présenter sous forme d'hypothèses $[\ldots]$ sur la structure innée d'un dispositif d'acquisition du langage. [...] Seront descriptivement adéquates les grammaires résultant de l'application des procédures postulées à une sélection de données suffisamment riche autrement dit, l'ensemble des procédures peut être considéré comme une hypothèse sur le système inné de l'acquisition linguistique ${ }^{4}$.

C'est ce que plusieurs chercheurs ont commencé à faire, notamment les dialectologues qui disposent d'une masse importante de données « réelles » permettant de tester les hypothèses élaborées trop souvent à partir d'un ou deux exemples issus de langues standard. De même, la syntaxe diachronique, peu développée jusqu'ici dans le cadre générativiste, connaît un essor croissant grâce à la constitution et l'utilisation de grands corpus, tant de textes écrits que de données dialectales qui témoignent d'états de langues successifs. C'est ainsi que grâce aux données du module morpho-syntaxique (MMS) du

4 Chomsky (1965 : 52-53) : "It is not, of course, necessary to assume that empiricist and rationalist views can always be sharply distinguished and that these currents cannot cross. [...] [They] can be presented as explicit hypotheses [...] about the innate structure of a languageacquisition device. [...] The grammars that result from application of the postulated procedures to a sufficiently rich selection of data will be descriptively adequate - in other words, [...] the set of procedures can be regarded as constituting an hypothesis about the innate languageacquisition system ». 


\section{OLIVIERI}

THESAURUS OCCITAN (THESOC) $)^{5}$, j'ai pu proposer une progression paramétrée de l'apparition des clitiques sujets dans les langues romanes ${ }^{6}$. En effet, si l'on considère que chaque dialecte est un état du jeu d'échecs de Saussure, on peut « lire » autrement la répartition géographique donnée par une carte linguistique, de manière à reconstruire la diachronie.

L'énorme avantage de ces grands corpus est alors qu'ils permettent d'étudier la variation linguistique, qu'elle soit diatopique ou diachronique, et ce, sans se limiter à une perspective typologique.

En effet, la variation intervient à différents niveaux, ce qui obscurcit les faits. Ainsi, la première variation est celle que tout le monde connaît, la variation interlinguistique, qui permet une classification typologique des langues du monde. Les paramètres utilisés dans cette perspective sont des «macroparamètres ${ }^{7}$, que les générativistes s'attachent à établir depuis le modèle GB.

Le second type de variation, qui est de plus en plus au cœur des analyses, est la microvariation qui rend compte de différences plus ténues, et que l'on observe entre des dialectes apparentés génétiquement. Mais il s'agit encore de variation interlinguistique, qui intervient entre des systèmes distincts, des langues certes proches mais différentes dans leurs structures. C'est ainsi que les syntacticiens qui étudient les dialectes en sont venus à proposer des «micro-paramètres », responsables de comportements plus subtils et plus fluctuants que ceux des langues standard, normées.

Mais il existe aussi une troisième variation, bien connue des dialectologues mais qui, bien qu'omniprésente dans le

5 Le THESOC est une base de données multimédias, couvrant l'ensemble des dialectes occitans et développée au sein de l'UMR 6039 «Bases, Corpus, Langage» (dir. J-Ph. Dalbera et M. Oliviéri) qui comporte, outre le lexique, des textes oraux étiquetés et analysés pour l'étude syntaxique. http://thesaurus.unice.fr. Cf. Brun-Trigaud \& Oliviéri (2009) et Georges (2005, à paraître).

6 Cf. Oliviéri (2009, 2010b). Il s'agit d'une progression en termes de traits, tels que [Personne], [Locuteur], [Genre] et [Nombre].

7 Cf. Kayne (1996). 
langage humain, n'a historiquement pas suffisamment été prise en compte par les syntacticiens, qui est la variation interne, intralinguistique. En général, elle a été considérée comme stylistique ou sociolinguistique mais n'a que peu fait l'objet d'études syntaxiques sérieuses. En réalité, les locuteurs d'une même langue n'utilisent pas tous les mêmes systèmes internes, ce que certains ont nommé «diglossie » ${ }^{8}$. La conséquence en est que tout un chacun est plurilingue, maîtrisant plusieurs systèmes linguistiques qui répondent à une logique interne, qu'il s'agit de décrire et de comprendre. La difficulté réside dans le flou qui entoure ces productions, la subjectivité des jugements de grammaticalité et la nécessité d'établir des critères précis discriminant ces différents systèmes, ces différents « lectes » ${ }^{9}$. Ainsi, Palasis (2009) a pu montrer, à partir de l'étude des clitiques sujets, la cohérence interne de chacun des deux «français » qu'elle définit, où les productions agrammaticales sont en distribution complémentaire, comme l'illustre l'exemple (3) :

$$
\begin{array}{ll}
\text { Français normé : } & \text { Jean dort. } \\
& \text { Il dort. } \\
& \text { Jean, il dort. } \\
\text { Français spontané : } & \begin{array}{cc}
\text { (a) Jean i-dort. } & \text { (b) *Jean il dort. } \\
\text { i-dort. } & \text { *Jean dort. } \\
& \text { *Il dort. }
\end{array}
\end{array}
$$

Cette perspective conduit alors à considérer avec attention la nature des faits soumis à l'analyse et donc ce qu'il convient de comparer afin de déterminer les principes universels et les paramètres à l'œuvre dans chacun des systèmes. Peut-on réellement mettre sur le même plan deux phrases issues de deux systèmes différents, sans prendre en compte l'existence de ces deux systèmes ${ }^{10}$ ? Ainsi il apparaît manifestement que les langues orales, spontanées, doivent être mises en regard avec

8 Cf. notamment Massot (2008), Zribi-Hertz (à paraître) et Palasis (2009).

9 Cf. Oliviéri (2010a : 58).

10 Comme l'affirme Scheer (2004: 58), «l'ambition générative à découvrir les universaux doit s'accompagner d'un contrôle structuraliste». 


\section{OLIVIERI}

d'autres systèmes oraux, de sorte que les dialectes peuvent être comparés avec le français oral, l'italien oral ou le portugais oral, mais pas avec les langues standard normées. Dans le cas contraire, on s'expose à des erreurs dans les jugements de grammaticalité, et donc dans les conclusions qu'on en tire.

\section{Présentation des articles}

Ce numéro consacré à la syntaxe de corpus se présente comme une sorte de panorama du genre. Les contributions présentées ici relèvent ainsi de différents modèles théoriques, abordent diverses questions, dans plusieurs langues et de plusieurs points de vue. Mais tous montrent l'importance de disposer d'un (grand) corpus, élaboré et construit par le chercheur, pour l'analyse des faits syntaxiques.

L'article de Katérina Palasis est une réflexion sur la construction d'un corpus destiné à l'étude de l'acquisition de la syntaxe. C'est une illustration directe de l'imbrication entre l'élaboration d'un corpus à partir d'enregistrements originaux et de la théorie, ici la grammaire générative. K. Palasis montre ainsi comment la nature des faits considérés par le passé a pu orienter, voire fausser les conclusions des analyses. Afin d'étudier le phénomène pro-drop chez les enfants français, K. Palasis constitue un corpus destiné à être intégré à la base de données CHILDES. Elle analyse précisément les différents problèmes liés à une telle entreprise et pose les principes qui président aux opérations nécessaires pour rendre le corpus pertinent et partageable par la communauté scientifique. L'analyse de ces données et la prise en compte de l'ensemble du système verbal des enfants la conduisent ainsi à reconsidérer les analyses antérieures concernant le paramètre du sujet nul en faisant de nouvelles propositions théoriques.

L'article de Maria-José Ezeizabarrena et Sandrine Aeby a également trait à l'acquisition, mais dans un contexte bilingue. S'inscrivant dans le cadre théorique générativiste, il traite du code-switching basque-espagnol dans des interactions adulte-enfant ou entre enfants dans un contexte bilingue. Les auteures explorent méthodiquement les différents types de code-switching basque-espagnol et mettent les analyses anté- 
rieures à l'épreuve des faits. Pour cela, elles présentent la constitution de leurs deux corpus, longitudinal et transversal, tant du point de vue méthodologique que du point de vue scientifique. L'analyse des données révèle la fragilité des propositions existant dans la littérature et montre que le codeswitching est soumis à des facteurs situés à l'interface syntaxe / pragmatique.

L'article d'Inés Fernández-Ordóñez est consacré à la présention d'un important corpus, le Corpus oral et sonore de l'espagnol rural (COSER) destiné à l'étude syntaxique et morpho-syntaxique des dialectes espagnols. I. FernándezOrdóñez y détaille la méthodologie et les fonctionnalités de ce corpus, montrant comment plusieurs phénomènes syntaxiques ont pu être élucidés grâce à cet outil. En effet, comparé aux atlas linguistiques antérieurs, le COSER permet de compléter les faits et de préciser les observations, tant en quantité qu'en qualité. Les analyses qui en proviennent apportent ainsi un nouveau point de vue et de nouvelles perspectives de recherche. Là encore, il s'agit de montrer comment des faits qui n'apparaissaient pas ou peu auparavant ne sont pas négligeables et forment en réalité un système cohérent.

L'article de Catarina Magro s'inscrit dans le cadre de la Morphologie distribuée et traite d'une particularité bien connue des dialectes portugais, l'interpolation. Son étude s'appuie sur un autre grand corpus, le Syntax-oriented Corpus of Portuguese Dialects - CORDIAL-SIN, ce qui lui permet de remettre en question des idées communément admises sur l'archaïsme de cette structure, et d'en montrer le caractère productif en portugais européen moderne. L'énorme avantage de l'utilisation d'un tel corpus réside dans la possibilité de confronter des données réelles aux hypothèses et prédictions des travaux antérieurs, menés sans recours à un corpus. En outre, ces données lui permettent d'apporter un autre point de vue sur un problème fort décrit, et de conclure avec de nouvelles propositions.

L'article de Franziska Hack traite de l'interrogation dans quelques dialectes ladins de l'Italie du Nord. Elle examine les données disponibles dans quelques corpus préexistants, tels 


\section{OLIVIERI}

qu'atlas et bases de données, en montrant les avantages et les inconvénients de ces sources. Cette étude l'amène à élaborer son propre corpus, à partir de nouvelles enquêtes dont elle justifie la méthodologie et les choix. En effet, son analyse se situant dans le cadre de la grammaire générative, l'objectif principal de l'élaboration de ce nouveau corpus est d'obtenir des données négatives et des jugements de grammaticalité, ce que ne fournissent pas les atlas. Ici encore, les présupposés théoriques du chercheur induisent les principes de l'enquête et régissent la construction du corpus qui, à son tour, permet l'analyse.

L'article de Leonardo M. Savoia et M. Rita Manzini, consacré également à certains dialectes du nord de l'Italie, réputés «occitans » ou «francoprovençaux », en examine le fonctionnement des clitiques sujets. Leur étude est fondée sur un important corpus élaboré à partir de leurs nombreuses enquêtes dialectales, qui prend en compte différents mécanismes concernant les clitiques sujets, tels que le redoublement $\mathrm{du}$ sujet lexical, l'interrogation ou l'interaction avec les clitiques objets. L'analyse minutieuse de ces données, menée dans le cadre de la grammaire générative, leur permet d'une part de confirmer et de préciser les hypothèses générativistes et d'autre part, d'effectuer une nouvelle partition dialectale, en contradiction avec des idées communément admises.

L'article de Sascha Gaglia est également consacré à l'analyse des clitiques sujets dans des dialectes italiens, ici ceux $\mathrm{du}$ Frioul. Inscrite dans le cadre de l'Optimality Theory, l'analyse repose sur une enquête orale axée sur des jugements de grammaticalité, complétée par un corpus biblique. En outre, S. Gaglia prend en compte dans son étude les données publiées et les résultats antérieurs (notamment Renzi \& Vanelli (1983) et Heap $(2000,2002))$. Considérant tous les cas d'omission du clitique sujet, il montre que ce phénomène est en relation avec la négation, les clitiques objets et les réfléchis. Il en propose une analyse en termes de contraintes consistant à éviter le syncrétisme.

L'article de Sandra Augendre, consacré à la dislocation en italien, touche à l'interface syntaxe / pragmatique. Son 
corpus est constitué de textes relevant d'un genre particulier, écrit mais informel, de type e-mail, chat et SMS. Elle en étudie les différents cas de dislocation, du sujet et de l'objet, à droite et à gauche et ce, d'un double point de vue, syntaxique et communicationnel. Au-delà de la classification distributionnelle des différents types de dislocation, son corpus et l'analyse qu'elle en fait lui permettent de proposer plusieurs hypothèses en termes de fonctions pragmatiques des structures syntaxiques. En effet, elle montre qu'il existe plusieurs types de dislocations, répondant à des fonctions différentes.

L'article de Christophe Benzitoun, Solène Bresson,

Laure Budzinski, Jeanne-Marie Debaisieux et Klara Holzheimer propose une étude distributionnelle de l'adjectif prochain en français fondée sur un important corpus diversifié. La moitié de ce corpus consiste en documents oraux tandis que l'autre est composée de textes écrits, ce qui permet aux chercheurs de comparer les deux systèmes, à la fois qualitativement et quantitativement. En outre, en confrontant leurs résultats à ceux de travaux menés à partir d'exemples fabriqués, ils montrent une fois de plus que l'étude de corpus apporte des indications que l'introspection ne permet pas de soupçonner. Cette analyse confirme encore l'importance de l'oral dans les études linguistiques, l'écrit élaboré ou les données des dictionnaires n'offrant qu' une vision partielle de la réalité linguistique.

L'article de Michael Zimmermann et Georg Kaiser se présente comme une étude induite par la préparation même de leur corpus. En effet, leur sujet d'étude, l'ancien français, les conduit à élaborer un corpus textuel annoté. Mais la simple annotation des données pose des questions d'ordre théorique que l'étude des faits permet à son tour d'éclaircir. En tentant d'établir le statut catégoriel des deux éléments et et ne, M. Zimmermann et G. Kaiser montrent ainsi comment le choix d'un modèle peut déterminer la mise en forme et l'enrichissement du corpus. D'autre part, après une confrontation minutieuse des faits aux conclusions des études antérieures, leur analyse des données leur permet de proposer une nouvelle conception du statut de ces éléments. 


\section{OLIVIERI}

On ne saurait, dans un seul numéro de Corpus, rendre compte de la diversité des approches de la syntaxe de corpus. Cependant, nous avons tenté d'en donner un échantillon, sinon homogène, du moins représentatif! Ce qui caractérise l'ensemble de ces contributions, c'est d'abord le besoin manifesté par les chercheurs de disposer de données en très grand nombre. A cette fin, de nombreuses bases de données informatiques voient le jour, de plus en plus élaborées, ce dont peut se réjouir l'ensemble de la communauté scientifique. Mais surtout, dans tous les cas, on voit ici que le corpus apparaît comme un «plus », un outil qui permet de renouveler la vision des faits. En effet, toutes ces études fondées sur corpus amènent les chercheurs à proposer de nouvelles analyses, à élaborer des hypothèses originales qui vont souvent à l'encontre de ce qui est généralement établi dans la littérature. En outre, la prise en compte de la performance, du E-language qui se manifeste dans les corpus, conduit également à approcher les interfaces de la grammaire, à s'interroger sur les relations que la syntaxe entretient avec la pragmatique ou la phonologie, ce qui semble très prometteur pour comprendre les mécanismes qui régissent le langage humain et appréhender l'architecture de la grammaire.

\section{Références}

Brun-Trigaud G. \& Oliviéri M. (2009). «Présentation du logiciel Thesaurus Occitan», in B. Horiot (éd.), La dialectologie hier et aujourd'hui (1906-2006). Lyon : Centre d'études linguistiques Jacques Goudet, 95-102.

Chomsky N. (1957). Syntactic Structures. La Haye : Mouton.

Chomsky N. (1965). Aspects of the Theory of Syntax. Cambridge, Massachussets : MIT.

Chomsky N. (2005). «Three Factors in Language Design», Linguistic Inquiry 36 (1) : 1-22.

Dalbera J.-Ph. (2002). « Le corpus entre données, analyse et théorie », Corpus $1:$ 89-104.

Georges P.-A. (2005). Réflexions linguistiques autour du développement de l'analyseur syntaxique du THESAURUS OCCITAN. Mémoire de Master, Université de Nice Sophia Antipolis. 
Georges P.-A. (à paraître). «The Thesaurus Occitan: a multimedia database dedicated to Occitan dialects. Presentation of its morphosyntax module », in J. L. Ormaetxea \& G. Aurrekoetxea (eds), Tools for Linguistic Variation (EUDIA-2). Bilbao: ASJU-ren gehigarriak, LIII, UPVEHU, 109-121.

Kayne R. S. (1996). «Microparametric syntax: Some introductory remarks », in J. R. Black \& V. Motapanyane (eds), Microparametric Syntax and Dialect Variation. Amsterdam : John Benjamins, ix-xviii.

Massot B. (2008). Français et diglossie. Décrire la situation linguistique française contemporaine comme une diglossie: arguments morphosyntaxiques. Thèse de Doctorat, Université de Paris 8.

Oliviéri M. (2009). « Typology or Reconstruction: the benefits of Dialectology for Diachronic Analysis », communication présentée à Going Romance XXIII. Nice, décembre 2009.

Oliviéri M. (2010a). A la recherche du clitique perdu... Habilitation à Diriger des Recherches, Université de Nice Sophia Antipolis.

Oliviéri M. (2010b). «From Dialectology to Diachrony: Evidence from Lexical and Morpho-Syntactic Reconstruction in Romance Dialects », in B. Heselwood \& C. Upton (eds), Proceedings of Methods XIII. Papers from The Thirteenth International Conference on Methods in Dialectology, 2008. Frankfurt : Peter Lang, 42-52.

Palasis K. (2009). «From Morphological Affixes to Syntactic Arguments: What Forces Children to Reinterpret Nominative French Clitics », communication présentée à Going Romance XXIII. Nice, décembre 2009.

Scheer T. (2004). «En quoi la phonologie est vraiment différente », Corpus 3 : 5-84.

Zribi-Hertz A. (à paraître). "Pour un modèle diglossique de description du français : quelques implications théoriques, didactiques et méthodologiques », JFLS 2011. 